\title{
Effect of glipizide treatment on postprandial lipaemia in patients with NIDDM
}

\author{
J. Jeppesen ${ }^{1}$, M.-Y.Zhou', Y.-D. I.Chen ${ }^{1,2}$, G.M. Reaven ${ }^{1,2}$ \\ ${ }^{1}$ Department of Medicine, Stanford University School of Medicine, Palo Alto, California, USA \\ ${ }^{2}$ Geriatric Research, Education and Clinical Center, Department of Veterans Affairs Medical Center, Palo Alto, California, USA
}

Summary The primary goal of the present study was to examine the effects of improved glycaemic control associated with glipizide treatment on postprandial lipaemia in non-insulin-dependent diabetic patients. The metabolism of triglyceride-rich lipoproteins of intestinal origin was assessed by measuring the retinyl palmitate content in plasma and the Svedberg flotation index (Sf) > 400 and Sf 20-400 lipoprotein fractions. Fasting plasma glucose concentrations $(14.5 \pm 0.5$ vs $9.0 \pm 0.5 \mathrm{mmol} / \mathrm{l}$ ), glycated haemoglobin levels (13.1 \pm 0.6 vs $9.7 \pm 0.6 \%$ ), and daylong plasma glucose concentrations were all significantly lower after glipizide treatment $(p<0.001)$. The improvement in glycaemic control was associated with increases in insulin-mediated glucose uptake $(p<0.001)$ and plasma post-heparin lipoprotein and hepatic lipolytic activities $(p<0.02)$. Both fasting plasma triglyceride ( $3.09 \pm 0.51$ vs $2.37 \pm$
$0.34 \mathrm{mmol} / \mathrm{l})$, and postprandial triglyceride concentrations $(p<0.05-0.001)$ were lower following glipizide treatment, associated with a significant fall in retinyl palmitate content in all three lipoprotein fractions $(p<0.02-0.001)$, with the most substantial decrease seen in the Sf 20-400 fraction. These data indicate that glipizide-induced improvement in glycaemic control was associated with changes in the metabolism of triglyceride-rich lipoproteins of intestinal origin that would be anticipated to reduce risk of coronary heart disease in non-insulin-dependent diabetic patients. [Diabetologia (1994) 37: 781-787]

Key words Intestinal lipoproteins, $\mathrm{Sf}>400$ fraction, $\mathrm{Sf}$ 20-400 fraction, insulin resistance, insulin suppression test, steady-state plasma glucose, steady-state plasma insulin.
The importance of CHD in individuals with NIDDM is clear [1]. However, commonly measured risk factors only account for a minor fraction of the excess CHD in NIDDM patients [1]. We have recently shown that postprandial concentrations of triglyceride-rich lipo-

Received: 25 November 1993

and in revised form: 11 March 1994

Corresponding author: G. M. Reaven, M.D., GRECC (182-B), VA Medical Center, 3801 Miranda Avenue, Palo Alto, CA 94304, USA

Abbreviations: RP, Retinyl palmitate; Sf, Sverdberg flotation index; $\mathrm{CHD}$, coronary heart disease; SSPI, steady-state plasma insulin concentration; SSPG, steady-state plasma glucose concentration; IDL, intermediate density lipoprotein; NEFA, non-esterified fatty acid; non-insulin-dependent diabetes mellitus. proteins of intestinal origin were higher in patients with NIDDM [2]. In 1979 Zilversmit [3] suggested that triglyceride-rich lipoproteins of intestinal origin, especially the cholesteryl ester-rich chylomicron remnants, could play a very important role in atherogenesis, and since then, several reports have been published supporting this hypothesis [4-10]. Thus, our observation that postprandial lipaemia was increased in NIDDM raised the possibility that abnormalities in the metabolism of intestinally-derived lipoproteins may contribute to the increased risk of CHD in these patients. Although we could not detect any correlation in our earlier paper between the magnitude of postprandial lipaemia and the level of glycaemic control, the crosssectional nature of the study protocol was not designed to carefully explore such a relationship. Consequently, we initiated the current study to see if improved glycaemic control associated with glipizide 
Table 1. Baseline characteristics of patients with NIDDM and control group (mean \pm SEM and range)

\begin{tabular}{lcc}
\hline & NIDDM & Control \\
$(n=18)$ & $(n=15)$ \\
\hline Age (years) & $56 \pm 2(35-72)$ & $61 \pm 2(41-72)$ \\
Gender (male/female) & $14 / 4$ & $10 / 5$ \\
BMI $\left(\mathrm{kg} / \mathrm{m}^{2}\right)$ & $26.9 \pm 0.6(20.6-30.7)$ & $27.3 \pm 0.5(24.5-30.5)$ \\
Waist/hip ratio & $0.94 \pm 0.01(0.83-1.04)$ & $0.91 \pm 0.02(0.76-1.03)$ \\
Triglyceride (mmol/l) & $2.37 \pm 0.34(0.72-7.22)$ & $2.24 \pm 0.24(1.14-5.01)$ \\
Systolic Blood Pressure (mmHg) & $140 \pm 2(122-162)$ & $142 \pm 4(101-160)$ \\
Diastolic Blood Pressure (mmHg) & $78 \pm 2(60-89)$ & $79 \pm 2(64-89)$ \\
Smoking (non-smoking/smoking) & $14 / 4$ & $15 / 0$ \\
Alcohol intake (oz/week) & $5 \pm 2(0-30)$ & $4 \pm 2(0-28)$ \\
Previous treatment: & & \\
Diet & 5 & --- \\
Sulfonylurea & 13 & -- \\
Insulin & 0 & --- \\
Duration of disease (years) & $8.3 \pm 1.9(0.1-28.0)$ & \\
\hline
\end{tabular}

treatment leads to a decrease in postprandial concentration of triglyceride-rich lipoproteins of intestinal origin in patients with NIDDM.

\section{Subjects and methods}

The study was approved by the Stanford Human Subjects Committee, and each research subject gave written, informed consent before entering the study. All 18 patients with NIDDM selected had a fasting plasma glucose concentration consistently above $11.1 \mathrm{mmol} / \mathrm{l}$ in the absence of specific anti-diabetic agents for at least 1 month prior to their enrollment in this study. The baseline characteristics of the study population are summarized in Table 1. All patients were in good general health, and taking no medication known to affect carbohydrate or lipoprotein metabolism. Data from a group of 15 healthy, non-diabetic subjects, selected to match the age, gender, BMI, and fasting plasma triglyceride concentrations of the patients with NIDDM are also presented for comparison. Subjects were admitted to the Stanford Clinical Research Center, and the following studies and laboratory analyses were performed after an overnight fast:

Resistance to insulin-mediated glucose disposal was determined by a modification [11] of the insulin suppression test [12]. This technique has been described in detail elsewhere [11]. Briefly, each subject received a continuous intravenous infusion of somatostatin $(5 \mu \mathrm{g} / \mathrm{min})$, insulin $\left(25 \mathrm{mU} \cdot \mathrm{m}^{2} \cdot \mathrm{min}^{-1}\right)$, and glucose $\left(0.67 \mathrm{mmol} \cdot \mathrm{m}^{-2} \cdot \mathrm{min}^{-1}\right.$ or $\left.120 \mathrm{mg} \cdot \mathrm{m}^{-2} \cdot \mathrm{min}^{-1}\right)$ via an indwelling teflon catheter in a superficial antecubital vein. Venous blood samples were obtained from a similar catheter inserted in a contralateral antecubital vein kept open with $0.9 \% \mathrm{NaCl}$ infusion containing $20 \mathrm{meq} / 1 \mathrm{KCl}$. The continuous infusion was given for $180 \mathrm{~min}$, and blood was obtained before and $30,60,90,120$, $150,160,170$, and 180 min after starting the infusion for measurement of plasma glucose and insulin. The mean value of the four measurements made during the last $30 \mathrm{~min}$ of the test was used to calculate the SSPI and SSPG. Given the similarity of the SSPI levels, the SSPG concentration provides a measure of insulin-mediated glucose disposal, i.e. the higher the SSPG, the more insulin resistant the subject; the lower the SSPG, the more insulin sensitive the subject.

Blood was obtained for measurement of fasting plasma triglyceride, cholesterol, and lipoprotein triglyceride and choleste- rol on three separate days.These samples were also subjected to sequential density ultracentrifugation [13] to separate VLDL, IDL, LDL, and HDL fractions at densities below 1.006, and at 1.019 and $1.063 \mathrm{~g} / \mathrm{ml}$, respectively, and the triglyceride and cholesterol contents of the various fractions were determined.

Postprandial carbohydrate and lipid metabolism were evaluated by serving subjects equicaloric test meals containing (as percent of total calories) $15 \%$ protein, $45 \%$ fat, and $40 \%$ carbohydrate. Subjects were given breakfast at 08.00 hours $(20 \%$ of daily calories) and lunch at 12.00 hours ( $40 \%$ of daily calories). After breakfast and lunch the subjects consumed only water or non-caloric decaffeinated beverages until the end of the study at 24.00 hours. Vitamin A (Aquasol; Astra Pharmaceutical Products, Inc., Westborough, Mass., USA), $60,000 \mathrm{U} / \mathrm{m}^{2}$ body surface area, in the form of retinyl alcohol, was given with lunch at noon. Blood for determination of retinyl ester, cholesterol, and triglyceride in plasma and various triglyceride-rich lipoprotein fractions was drawn into tubes containing EDTA and $120 \mu \mathrm{mol} / \mathrm{I}$ butylated hydroxytoluene at hourly intervals from 12.00 to 18.00 hours and every $2 \mathrm{~h}$ from 18.00 to 24.00 hours. The absorbed vitamin A, retinyl alcohol, is esterified in intestinal cells, and the retinyl ester incorporated in newly-synthesized intestinal lipoproteins and secreted [14]. Thus, the esterified retinyl ester provides a technique for identifying and quantifying chylomicron and chylomicron remnants [15]. All samples for retinyl ester determination were shielded from light with aluminum foil. Three millilitres of fresh plasma from each time point were overlayed with $2.2 \mathrm{ml} 0.9 \% \mathrm{NaCl}$ and ultracentrifuged for $100,000 \mathrm{~g}$ $\times 44 \mathrm{~min}$ at density $<1.006 \mathrm{~g} / \mathrm{ml}$ and $15^{\circ} \mathrm{C}$ in a 50.3 rotor using $20,000 \mathrm{rev} / \mathrm{min}$ to float lipoprotein particles of Sf $>400$. The infranatant from the original separation was overlayed with $1.15 \%$ $\mathrm{KBr}$ and subjected to ultracentrifugation using the same rotor with $39,000 \mathrm{rev} / \mathrm{min}$ at density $=1.006 \mathrm{~g} / \mathrm{ml}$ and $10^{\circ} \mathrm{C}$ for $100,000 \mathrm{~g} \times 15 \mathrm{~h}$, and the top layer obtained defined as the $\mathrm{Sf} 20$ 400 fraction and the infranatant defined as the $\mathrm{Sf}<20$ fraction. Samples were processed as quickly as possible in the laboratory under subdued light. Sf denotes Sverdberg flotation index, an operational index calculated from the density, viscosity of plas$\mathrm{ma}$, and the total centrifugation force $(g \times$ time $)$ necessary to float a particle to the top by analytical ultracentrifugation. The higher the Sf value, the more bouyant the particle, and the greater its triglyceride content. In the postprandial state, the Sf $>400$ lipoprotein fraction contains predominantly chylomicrons, whereas the Sf 20-400 lipoprotein fraction contains VLDL particles as well as chylomicron remnants [16]. 

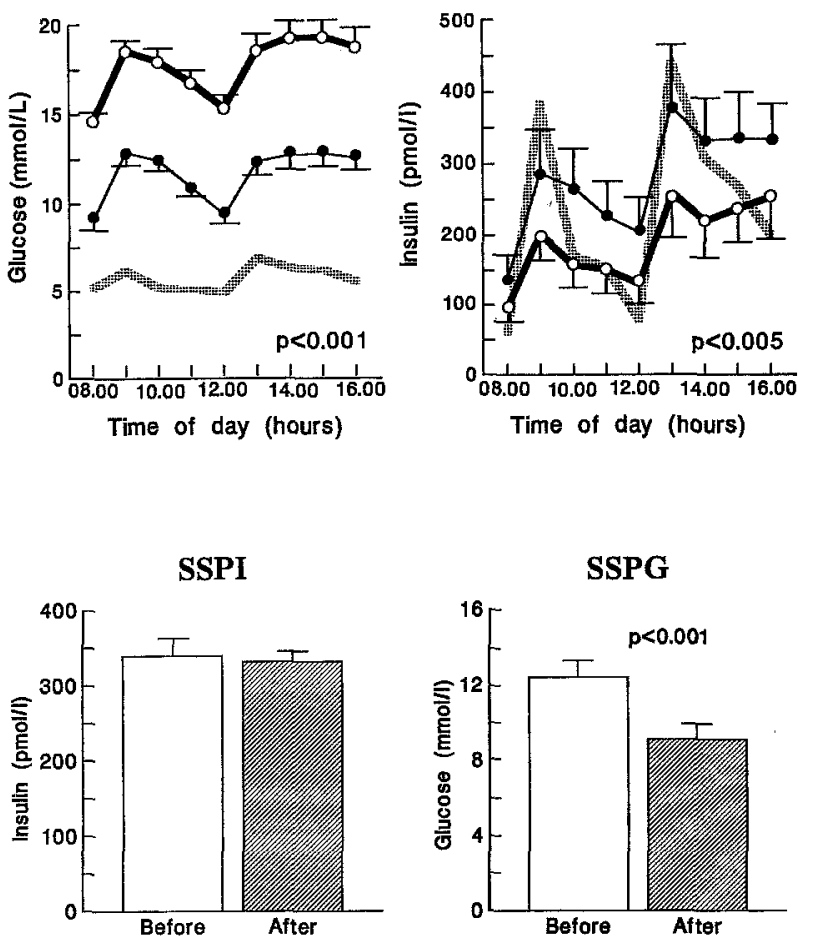

Fig. 2. Steady-state plasma insulin (SSPI, left) and glucose (SSPG, right) concentrations during modified insulin suppression tests in patients with NIDDM before ( $\square$ ) and after (因) glipizide treatment

Table 2. Fasting lipid and lipoprotein concentrations ( $\mathrm{mmol} / \mathrm{l}$ ) before and after glipizide treatment

\begin{tabular}{llll}
\hline Variable & Before & After & $p$ \\
\hline Cholesterol & $4.91 \pm 0.17$ & $4.73 \pm 0.15$ & $<0.05$ \\
VLDL-cholesterol & $1.40 \pm 0.23$ & $1.06 \pm 0.17$ & $<0.05$ \\
IDL-cholesterol & $0.22 \pm 0.03$ & $0.28 \pm 0.04$ & $<0.05$ \\
LDL-cholesterol & $2.27 \pm 0.15$ & $2.32 \pm 0.13$ & $\mathrm{NS}$ \\
HDL-cholesterol & $1.03 \pm 0.05$ & $1.07 \pm 0.05$ & $<0.02$ \\
Chol/HDL-cholesterol & $4.91 \pm 0.28$ & $4.53 \pm 0.20$ & $<0.01$ \\
Triglyceride & $3.09 \pm 0.51$ & $2.37 \pm 0.34$ & $<0.05$ \\
VLDL-Triglyceride & $2.61 \pm 0.50$ & $1.89 \pm 0.33$ & $<0.05$ \\
IDL-Triglyceride & $0.12 \pm 0.01$ & $0.12 \pm 0.01$ & $\mathrm{NS}$ \\
LDL-Triglyceride & $0.22 \pm 0.01$ & $0.21 \pm 0.01$ & $\mathrm{NS}$ \\
HDL-Triglyceride & $0.14 \pm 0.01$ & $0.14 \pm 0.01$ & $\mathrm{NS}$ \\
\hline
\end{tabular}

Aliquots of plasma, Sf $>400$, Sf $20-400$, and Sf $<20$ lipoprotein fractions were extracted by chloroform/methanol $=2: 1$ (Folch's solution) using HPLC-grade solvent. A known quantity of retinyl acetate (250-500 ng) was added to each sample before extraction as an internal standard. Extracted material was dried under a nitrogen stream, reconstituted in Folch's solution, and separated and quantitated on HPLC at $326 \mathrm{~nm}$, using a reverse phase Supelcosil LC-8 column, $25 \mathrm{~mm} \times 4.6 \mathrm{~mm}$ internal diameter, (Supelco Inc., Bellefonte, Pa., USA) with $100 \%$ methanol as the mobile phase at a flow rate of $1.75 \mathrm{ml} / \mathrm{min}$ to separate retinyl alcohol and retinyl ester. Since $80 \%$ of the retinyl ester is represented by RP, all results are presented as RP concentrations. Standard curves were created for RP with the concentrations of this compound being calculated by using a molar extinction coefficient of 52275 at $326 \mathrm{~nm}$. The inter-assay coefficient of variance of plasma RP in our laboratory is $8 \%$. Isolated lipopro-

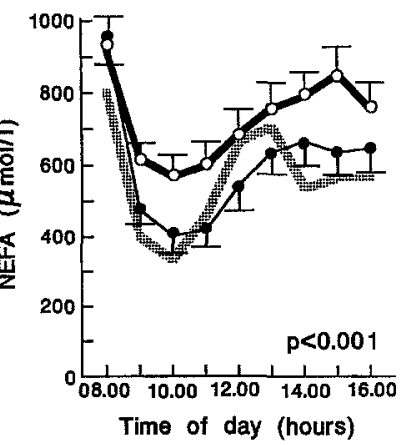

Fig.1. Plasma glucose (left), insulin (middle), and NEFA (right) concentrations in patients with NIDDM before (O) and after $(\bullet)$ glipizide treatment. Control group is represented by shaded curve. Standard error of the control group was omitted for clarity tein fractions have a smaller inter-assay variance of $5 \%$. The intra-assay variance of ten repeated injections of retinyl acetate is $2 \%$, as we have demonstrated previously [2]. When vitamin A is given with lunch at noon, $4 \mathrm{~h}$ after a standard breakfast, the peak appearance of RP in plasma occurs $2-4 \mathrm{~h}$ post-lunch, and the majority of RP is present in the $\mathrm{Sf}>400$ fraction throughout most of the $10-\mathrm{h}$ period of measurement with very little RP present in the Sf $<20$ fraction until the end of the study (the 12 th $h$ ). $\mathrm{RP}$ concentrations in Sf $>400$ and Sf $20-400$ fractions were measured directly, as well as by calculating the differences between the RP concentrations measured in plasma and $\mathrm{Sf}<400$ (infranatant of plasma free of Sf $>400$ particles) and that in Sf $<400$ and Sf $<20$ (infranatant of plasma free of both $\mathrm{Sf}>400$ and Sf 20-400 particles, respectively). The mean values of direct and indirect estimate of RP concentrations were presented.

In addition, blood was drawn into tubes containing EDTA before breakfast and at hourly intervals from 08.00 to 16.00 hours for measurement of glucose, insulin, and NEFA concentrations.

Plasma hepatic lipase activity HLA was measured as follows: on separate days, each subject was given an intravenous bolus injection of heparin $(100 \mathrm{unit} / \mathrm{kg})$ to release lipases into the circulation. Blood was drawn before, 30 and 45 min later. Lipase activity was maximal at $30-\mathrm{min}$, and remained plateaued at $45 \mathrm{~min}$, and the results presented were determined in the 30 -min postheparin samples. $\mathrm{A}^{3} \mathrm{H}$-triolein emulsion was prepared according to Nilsson-Ehle and Ekman [17] with a final triglyceride concentration of $2 \mathrm{mmol} / 1$ and $50-100 \mu \mathrm{l}$ of a six-fold diluted postheparin plasma was added to $100 \mu \mathrm{l}$ of ${ }^{3} \mathrm{H}$-triglyceride emulsion and incubated for $30 \mathrm{~min}$ at $37^{\circ} \mathrm{C}$. The released ${ }^{3} \mathrm{H}-\mathrm{NEFA}$ was extracted and separated from ${ }^{3} \mathrm{H}$-triglyceride substrate as described by Belfrage and Vaughan [18], and radioactivity assessed by scintillation spectrometer. In order to distinguish the activity of post-heparin lipoprotein lipase from that of hepatic lipase, a high salt concentration $(1 \mathrm{~mol} / \mathrm{l} \mathrm{NaCl})$ was employed in a separate incubation to inhibit lipoprotein lipase, so that only hepatic lipase activity was measured. The lipoprotein lipase activity was then determined by the difference between the total lipolytic activity and the hepatic activity. The results are expressed as $\mu \mathrm{mol}$ NEFA released per h per ml of post-heparin plasma. The coefficients of variation for the measurements of the lipoprotein and hepatic lipase assays were $4.3 \%$ and $4.2 \%$, respectively.

Concentrations of glucose [19] triglyceride [20], cholesterol [21], and NEFA [22], were measured enzymatically, and insulin was determined by RIA [23]. The concentration of glycated haemoglobin was measured by microaffinity chromatography column [24].

After the outlined series of tests, patients were started on glipizide treatment and discharged to outpatient follow-up. Patients were seen at weekly intervals, and the glipizide dose titrated until either excellent glycaemic control was achieved, defined as a fasting plasma glucose concentration less than 

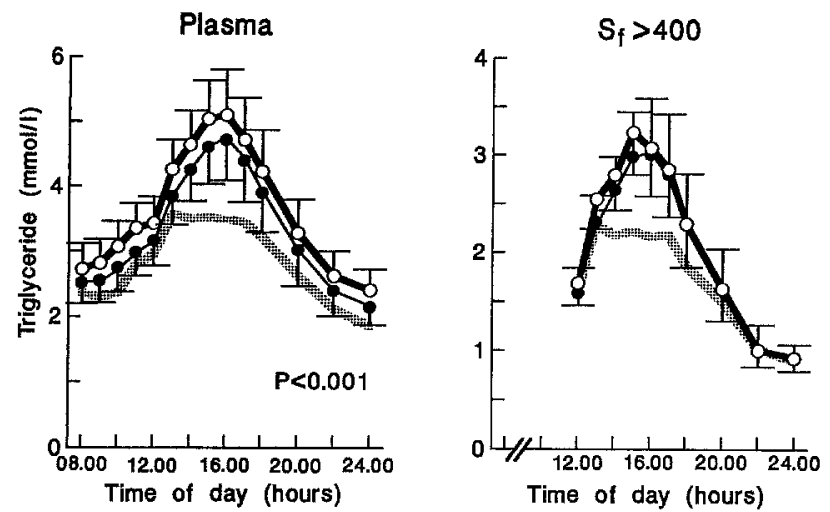

$7.8 \mathrm{mmol} / \mathrm{l}$, or the maximum dose of glipizide had been reached, defined as $20 \mathrm{mg}$ twice daily. The patients were then re-admitted to the Clinical Research Center after at least 8 weeks $(9 \pm 1.4)$ of glipizide treatment, and the measurements which had been done at baseline repeated. The mean glipizide dose was $36.4 \pm 7.5 \mathrm{mg}$, and all patients were taking glipizide two times daily, one dose each before breakfast and dinner. Glipizide treatment was continued as usual during the second admission. On the day of meal test, glipizide was only given before breakfast, since the research subjects were fasting after lunch until midnight. Diet composition and exercise were not altered during the treatment period, but caloric intake was modified as necessary to prevent weight gain associated with improved glycaemic control. Weight did not change $(83.4 \pm 10.2 \mathrm{~kg}$ before and $83.9 \pm 10.7 \mathrm{~kg}$ after) during the glipizide treatment period, and no significant side-effects were reported.

\section{Statistical analysis}

Variables are expressed as the mean \pm SEM, and statistical evaluation was performed with the Statistical Analysis System program (SAS Institute, Cary, N.C., USA) using generalized linear models procedure. To evaluate the effect of glipizide treatment, values before and after treatment were compared by either Student's paired $t$-test or three-way analysis of variance (ANOVA) with "patient", "effect" and "time" being the three factors [25]. Multiple regression coefficients were also calculated with the Statistical Analysis System program [26].

\section{Results}

Plasma glucose, insulin, and NEFA concentrations between 08.00-16.00 hours are shown in Figure 1. After glipizide treatment, overall glycaemic control improved substantially. Fasting plasma glucose concentrations were significantly lower $(9.0 \pm 0.5$ vs $14.5 \pm 0.5 \mathrm{mmol} / \mathrm{l} ; p<0.001$ ), and there was a highly significant decrease in daylong plasma glucose concentrations following treatment $(p<0.001$, three-way ANOVA). However, glipizide-treated patients remained very hyperglycaemic compared to the control group shown in the shaded curve. Improved glycaemic control following glipizide treatment was reflected by a significant fall in the concentration of glycated haemo-

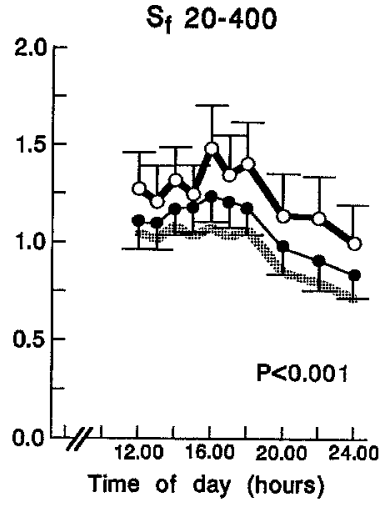

Fig.3. Triglyceride concentrations in plasma (left) and in the $\mathrm{Sf}>400$ (middle) and Sf 20-400 (right) lipoprotein fractions in patients with NIDDM before ( $O$ ) and after $(\bullet)$ glipizide treatment and the control group (O). Control group is represented by shaded curve globin from $13.1 \pm 0.6$ to $9.7 \pm 0.6 \%(p<0.001)$. It can also be seen from Figure 1 that plasma insulin concentrations between $08.00-16.00$ hours were significantly higher $(p<0.005)$ after glipizide treatment in patients with NIDDM. Finally, the results in the right panel of Figure 1 show that glipizide treatment was associated with significantly lower daylong NEFA concentrations $(p<0.001)$, which were now similar to the control values.

The results of the measurements of insulin resistance by the insulin suppression test are shown in Figure 2. It is apparent from the left panel of Figure 2 that SSPI was similar in patients with NIDDM before and after glipizide treatment. However, it can be seen from the right panel of Figure 2 that SSPG concentrations were $38 \%$ lower $(9.1 \pm 0.8$ vs $12.4 \pm 0.9 \mathrm{mmol} / \mathrm{l}$, $p<0.001$ ) in patients with NIDDM following treatment with glipizide.

Fasting plasma lipid and lipoprotein concentrations before and after glipizide treatment are shown in Table 2. There results indicate that total cholesterol concentrations were significantly lower $(p<0.05)$ after glipizide treatment, predominantly due to a fall in VLDL-cholesterol $(p<0.05)$. In contrast, HDL-cholesterol was significantly higher $(p<0.02)$ following glipizide administration. Consequently, the ratio of total to HDL-cholesterol decreased from $4.91 \pm 0.28$ to $4.53 \pm 0.20(p<0.01)$. Fasting plasma triglyceride concentrations were reduced $(p<0.05)$ following glipizide treatment, and, as with total cholesterol, this change was due to a decrease in VLDL-triglyceride $(p<0.05)$.

Triglyceride concentrations from 08.00 to 24.00 hours in plasma, and from 12.00 to 24.00 hours in the $\mathrm{Sf}$ $>400$ and Sf $20-400$ lipoprotein fractions, are seen in Figure 3. Following glipizide treatment, postprandial plasma triglyceride concentrations were somewhat lower than before treatment $(p<0.001)$, as were the triglyceride concentrations in the Sf 20-400 fraction $(p<0.001)$. However, triglyceride concentrations did not change significantly in the $\mathrm{Sf}>400$ lipoprotein fraction. Values for a control group with comparable fasting triglyceride concentrations are included in this figure for the sake of comparison. 

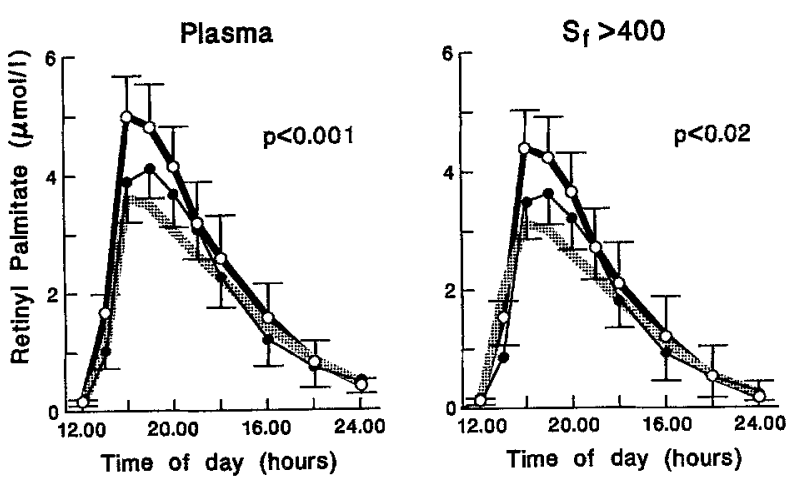

Lipoprotein lipase
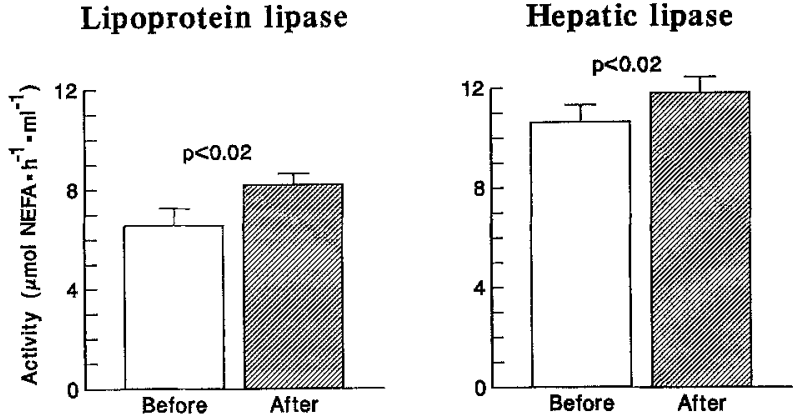

Fig.5. Plasma post-heparin lipoprotein lipase (left) and hepatic (right) lipase activities in patients with NIDDM before ( $\square$ ) and after ( ) glipizide treatment

The concentration of triglyceride-rich lipoproteins of intestinal origin as assessed by RP concentrations in plasma and the Sf $>400$ and Sf $20-400$ fractions are shown in Figure 4. These results indicate that there were significant decreases $(p<0.001-<0.02)$ in RP concentrations following glipizide treatment in total plasma and in both lipoprotein fractions. It can also be seen that the RP concentrations following glipizide therapy returned towards the values seen in the reference control group.

Figure 5 shows that both post-heparin lipoprotein lipase and hepatic lipase activities were significantly higher $(p<0.02)$ in association with glipizide treatment.

The results presented to this point have shown that significant changes in postprandial plasma glucose, insulin, NEFA, and triglyceride and triglyceride-rich intestinal lipoprotein concentrations were seen following glipizide treatment in patients with NIDDM, associated with enhanced insulin-mediated glucose disposal and higher levels of lipoprotein and hepatic lipase activities. In an effort to define the relationship between these changes, multiple regression coefficients were calculated. When this was done, approximately $25 \%$ of the treatment effects on lipid metabolism could be accounted for by the changes in plasma glucose, insulin and NEFA concentrations, insulin-mediated glucose disposal, and lipase activity, and none of these variables, by itself, was significantly correlated with the

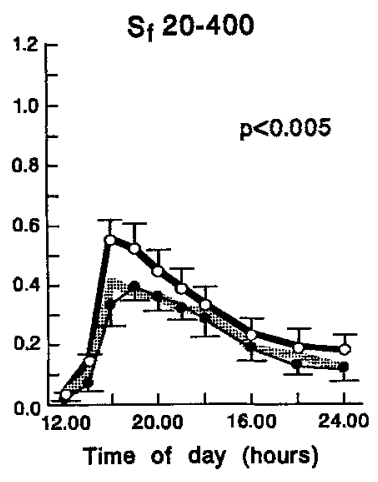
Fig.4. Retinyl palmitate concen- trations in plasma (left) and in the Sf $>400$ (middle) and Sf 20-400 (right) lipoprotein fractions in pa- tients with NIDDM before $(O)$ and after $(\bullet)$ glipizide treatment. Control group is represented by shaded curve

changes in lipoprotein metabolism (data not shown). Thus, we are not able to propose any specific cause and effect relationship to account for the improvement in lipoprotein metabolism seen in association with glipizide treatment.

\section{Discussion}

The results of this study showed that glycaemic control improved following initiation of glipizide treatment, and that the decrease in ambient glucose concentration was associated with lower levels of glycated haemoglobin, an increase in ambient daylong insulin concentrations, a decrease in daylong NEFA concentrations, and enhanced insulin-mediated glucose disposal. All of these changes have been previously demonstrated to occur with glipizide treatment of patients with NIDDM $[27,28]$.

In addition to the better glycaemic control, as well as improvements in estimates of insulin secretory response and insulin action, glipizide treatment was also associated with beneficial changes in lipid and lipoprotein metabolism. Specifically, total and VLDL-cholesterol were lower and HDL-cholesterol higher following glipizide administration. As a consequence, the ratio of total to HDL-cholesterol was lower in glipizide-treated patients. In addition, plasma triglyceride and VLDL-triglyceride concentrations decreased in association with glipizide treatment. This finding is similar to results of earlier studies of sulphonylurea treatment of patients with NIDDM [29-31], and seems to be related to both a decrease in hepatic VLDL-triglyceride secretion and an increase in rate of removal of VLDL-triglyceride from plasma. It is tempting to speculate that the former is related to the observed fall in ambient NEFA concentrations, and the latter to the increase in lipase activity.

Postprandial concentrations of triglyceride-rich lipoproteins were also lower in association with glipizide treatment, particularly when intestinal lipoproteins were specifically quantified. Thus, RP concentrations from 12.00-24.00 hours were lower in plasma and in both the Sf $>400$ and Sf $20-400$ lipoprotein fractions. 
Although, all of these changes were statistically significant, the greatest difference was seen in the Sf $20-400$, or chylomicron remnant fraction. It should be noted that plasma post-heparin lipoprotein lipase activity was also higher after glipizide treatment, and this change may have contributed to the decrease in concentration of triglyceride-rich lipoproteins in glipizide-treated subjects.

These results are of significant clinical relevance in that they demonstrate that the previously defined defect in the metabolism of triglyceride-rich lipoproteins in patients with NIDDM [2] can be at least partially reversed when glycaemic control is improved with glipizide. There is considerable evidence indicating that plasma triglyceride concentrations are higher than normal in patients with NIDDM [32], and that this change is associated with increased risk of CHD in these patients $[33,34]$. However, the link between a high plasma triglyceride concentration and CHD is far from obvious. For example, patients with NIDDM and/or hypertriglyceridaemia have been shown to have increases in plasminogen activator inhibitor-1 $[35,36]$, smaller, denser LDL particles [37, 38], and increased postprandial lipaemia $[2,39]$; changes which have been proposed as increasing the risk of CHD in non-diabetic subjects [3-9]. It seems reasonable to speculate that a similar relationship between these changes and $\mathrm{CHD}$ would also exist in patients with NIDDM. Consequently, the increased postprandial concentrations of triglyceride-rich lipoproteins of intestinal origin shown to be present in patients with NIDDM $[2,39]$ may well contribute to the excess of CHD in these patients. If this is the case, it is obvious that the decrease in the concentration of these potentially atherogenic lipoproteins associated with glipizide treatment is of significant clinical importance. On the other hand, it should be emphasized that the improvements in lipoprotein metabolism are not necessarily due to glipizide, per se, and might occur in response to improved glycaemic control with any sulphonylurea compound. Indeed, it could be argued that the changes in lipoprotein metabolism are independent of the method used to lower blood glucose. Obviously, these are issues that can only be resolved by additional studies.

Acknowledgements. This research was supported by research grants from the National Institutes of Health (HL-08506 and RR00070). Dr. Jeppesen is supported by a fellowship from Novo-Nordisk, Bagsvaerd, Denmark.

\section{References}

1. Pyörälä K, Laakso M, Uusitupa M(1987) Diabetes and atherosclerosis: an epidemiologic view. Diabetes Metab Rev 3: 463-524

2. Chen Y-DI, Swami S, Skowronski R, Coulston A, Reaven GM (1993) Differences in postprandial lipemia between pa- tients with normal glucose tolerance and noninsulin-dependent diabetes mellitus. J Clin Endocrinol Metab 76: 172-177

3. Zilversmit DB (1979) Atherogenesis: a postprandial phenomenon. Circulation 60: 473-485

4. Stender S, Zilversmit DB (1981) Arterial influx of esterified cholesterol from two plasma lipoprotein fractions and its hydrolysis in vivo in hypercholesterolemic rabbits. Atherosclerosis 39: 97-109

5. Stender S, Zilversmit DB (1982) Comparison of cholesteryl ester transfer from chylomicrons and other plasma lipoproteins to aorta intima-media of cholesterol-fed rabbits. Arteriosclerosis 2: 493-499

6. Simons LA, Sweyer T, Simons J et al. (1987) Chylomicrons and chylomicron remnants in coronary artery disease: a casecontrol study. Atherosclerosis 65: 181-189

7. Simpson HS, Williamson CM, Olivecrona T (1990) Postprandial lipemia, fenofibrate and coronary artery disease. Atherosclerosis $85:$ 193-202

8. Groot PHE, van Stiphout WAHJ, Krauss XH (1991) Postprandial lipoprotein metabolism in normolipidemic men with and without coronary atherosclerosis. Arterioscler Thromb 11: 653-662

9. Patsch JR, Miesenböck G, Hopferwieser T et al. (1992) Relation of triglyceride metabolism and coronary artery disease: studies in the postprandial state. Arterioscler Thromb 12: $1336-1345$

10. Karpe F, Bard JM, Steiner G, Carlson LA, Fruchart JC, Hamsten A (1993) HDLs and alimentary lipemia: studies in men with previous myocardial infarction at young age. Arterioscler Thromb 13: 11-22

11. Shen D-C, Shieh S-M, Fuh M-T, Wu D-A, Chen Y-DI, Reaven GM (1988) Resistance to insulin-stimulated-glucose uptake in patients with hypertension. J Clin Endocrinol Metab 66: 580-583

12. Shen S-W, Reaven GM, Farquhar J (1970) Comparison of impedance to insulin-mediated glucose uptake in normal subjects and in subjects with latent diabetes. J Clin Invest 49: $2151-2160$

13. Havel RJ, Eder HA, Bragdon JH (1955) The distribution of ultracentrifugally separated lipoproteins in human serum. $\mathrm{J}$ Clin Invest 34: 1345-1353

14. Blomhoff R, Green MH, Green JB, Berg T, Norum KR (1991) Vitamin A metabolism: new perspectives on absorption, transport, and storage. Physiol Rev 71(4): 951-990

15. Hazzard WR, Bierman EL (1976) Delayed clearance of chylomicron remnants following vitamin A-containing oral fat loads in broad- $\beta$ disease (type III hyperlipoproteinemia). Metab Clin Exp 25: 777-801

16. Chen Y-DI, Reaven GM (1991) Intestinally-derived lipoproteins: metabolism and clinical significance. Diabetes/Metab Rev 7: 191-208

17. Nilsson-Ehle P, Ekman R (1977) Rapid, simple and specific assays for lipoprotein lipase and hepatic lipase. Artery 3: 194-209

18. Belfrage $P$, Vaughan M (1969) Simple liquid-liquid partition system for isolation of labeled oleic acid from mixtures with glyceride. J Lipid Res 10: 341-344

19. Kadish AH, Litle RH, Sternberg JC (1968) A new and rapid method for determination of glucose by measurement of rate of oxygen consumption. Clin Chem 14: 116-131

20. Wahlefeld AW (1974) Triglycerides. Determination after enzymatic hydrolysis. In: HU Bergmeyer (ed) Methods of enzymatic analysis. Academic Press, New York, pp 18311835

21. Allain CA, Poon LS, Chun CSG, Richmond W, Fu PC (1974) Enzymatic determination of total serum cholesterol. Clin Chem 20: $470-475$ 
22. Noma A, Okabe H, Kita M (1973) A new colorimetric microdetermination of free fatty acids in serum. Clin Chim Acta 43: $317-320$

23. Hales CN, Randle PJ (1968) Immunoassay of insulin with insulin antibody precipitate. Biochem J 88: 137-139

24. Abraham EC, Perry RE, Stallings M (1983) Applications of affinity chromatography for separation and quantitation of glycosylated hemoglobins. J Lab Clin Med 102:187-197

25. Winer BJ (1972) Design and analysis of factorial experiments: nested factors (hierarch designs). In: Winer BJ (ed) Statistical principles in experimental design. McGraw-Hill, New York, pp 359-366

26. Myers RH (1986) Classical and modern regression with applications. Duxbury, Boston, MA

27. Greenfield MS, Dobhern L, Rosenthal M, Schulz B, Widstrom A, Reaven GM (1982) Effect of sulfonylurea treatment on in vivo insulin secretion and action in patients with non-insulin-dependent diabetes mellitus. Diabetes 31: 307312

28. Jeng C-Y, Hollenbeck CB, Wu MS, Foley JE, Chen Y-DI, Reaven GM (1991) Changes in carbohydrate metabolism in association with glipizide treatment of type 2 diabetes. Diabet Med 8: 37-39

29. Greenfield MS, Doberne L, Rosenthal M, Vreman HJ, Reaven GM (1982) Lipid metabolism in non-insulindependent diabetes mellitus: effect of glipizide therapy. Arch Intern Med 142: 1498-1500

30. Dunn FL, Raskin P, Bilheimer DW, Grundy SM (1984) The effect of diabetic control on very low-density lipoproteintriglyceride metabolism in patients with type II diabetes mellitus and marked hypertriglyceridemia. Metabolism 33: 117120

31. Taskinen MR, Beltz WF, Harper I et al. (1986) Effects of NIDDM on very-low-density lipoprotein triglyceride and apolipoprotein B metabolism. Diabetes 35: 1268-1277
32. Reaven GM, Chen Y-DI (1988) Role of insulin in regulation of lipoprotein metabolism in diabetes. Diabetes Metab Rev 4: 639-652

33. West KM, Ahuja MMS, Bennett PH et al. (1983) The role of circulating glucose and triglyceride concentrations and their interactions with other "risk factors" as determinants of arterial disease in nine diabetic population samples from the WHO multinational study. Diabetes Care 6:361-369

34. Fontbonne A, Eschwège E, Cambien F et al. (1989) Hypertriglyceridaemia as a risk factor of coronary heart disease mortality in subjects with impaired glucose tolerance or diabetes. Diabetologia 32: 300-304

35. Juhan-Vague I, Roul C, Alessi MD, Ardissone JP, Heim M, Vague $P$ (1989) Increased plasminogen activator inhibitor activity in non-insulin dependent diabetic patients. Relationship with plasma insulin. Thromb Haemast 61:370-373

36. Juhan-Vague I, Alessi MC, Vague P (1991) Increased plasma plasminogen activator inhibitor 1 levels. A possible link between insulin resistance and atherothrombosis. Diabetologia 34: 457-462

37. Suzuki N, Oikawa S-I, Hori S et al. (1989) Appearance of multidisperse low density lipoprotein and altered lipoprotein composition in non-insulin-dependent diabetes with type IIa hyperlipoproteinemia. Metabolism 38: 224-229

38. Feingold KR, Grunfeld C, Pang M, Doerrler W, Krauss RM (1992) LDL subclass phenotypes in non-insulin-dependent diabetes. Arterioscler and Thromb 12: 1296-1502

39. Lewis GF, O'Meara NM, Soltys PA et al. (1991) Fasting hypertriglyceridemia in noninsulin-dependent diabetes mellitus is an important predictor of postprandial lipid and lipoprotein abnormalities. J Clin Endocrinol Metab 72: 934-944 